\title{
Dynamic Simulation of Deposition Processes of Spacecraft Molecular Contamination
}

\author{
Jia QIAO*, Shengsheng YANG, Jianjun LI, Xing GUO, Yi WANG
}

\begin{abstract}
Accurate simulation and calculation of the deposition of outgassing molecule can shorten the cycle and reduce the cost of vacuum tests on satellites. It also provides a reference for contamination protection design by systems engineers. In this study, the molecular outgassing, transport and deposition processes were simulated by diffusion theory, the angle coefficient method, and the first-order desorption equation, respectively. The simulation results were consistent with the test data trends, but deviated from the test values. Given the effect of initial molecular outgassing rate, diffusion coefficient and residence time on the deposition mass, it was surmised that considering the molecular species and the weight mass rate would improve the calculation result. These considerations indeed improved the numerical simulations of highvacuum contamination.
\end{abstract}

Keywords: angle coefficient method; diffusion theory; first order desorption

\section{INTRODUCTION}

In the space environment, the organic materials used in satellites are outgassed, diffused, and deposited on the surfaces of optical elements or thermal control devices, where they degrade the performance of the elements or coatings. The degree of contamination depends on the position of the contaminated surface. To alleviate the contamination effect, engineers assess the outgassing performance of materials. The ASTM E 1559 standard [1] measures the outgassing, deposition, outgassing rate and deposition rate in situ and in real-time. Testers usually check the material outgassing with equipment based on this standard. However, the test is costly and time consuming. Moreover the desire to perform sample return as well as studies on the composition of exospheres of moons and the coma of comets has driven improvements in instrument performance [2-6]. To resolve this problem, the present study simulates the molecular contamination produced during a vacuum test of two materials. The contamination is predicted by a virtual test which shortens the cycle and reduces the cost of the real test, and provides a reference for the design of satellites and selection of materials. Moreover, the virtual test technology can predict contamination depositions that cannot be measured physically.

Since the 1970s, the effect of material outgassing contamination has been extensively researched. Wong, Labatete-Goeppinger, Fowler and others [7-9] simulated the deposition of contaminant molecules bouncing in a hypothetical cavity in COMSOL Multiphysics software. They found that large openings provide venting pathways that dispel the contaminants into space. These contaminant molecules tend to settle on colder surfaces. Khassanchine, Grigorevskiy, and Galygin [10-12] analyzed the spacetime distribution of the molecules outgassed from materials and the time distribution of the mass deposited on a unit surface. Yuan, X. X. et al. [13] studied the molecular contamination transport process, the deposition of the molecular contamination on the satellite solar cell and the attenuation of the solar cell output power during barbital operation by direct simulation monte carlo (DSMC), but they did not analyze the relation deposition distribution with time, nor verify with experiment. Jin, X. H. et al. [14] studied the molecular return flux problem by Test Particle
Monte Carlo (TPMC). They analyzed the impact of geometric surfaces, geometric radius, the velocity of the incoming flow, the number density of the incoming flow and the angle of attack of the incoming flow on return flux. They found the results obtained by TPMC method were in keeping with DSMC results, and TPMC needed shorter computer time. But they did not give the relationship between deposition and time, nor verify with experiment. Shuang Wei [15] described the gas outflow mechanism of the materials, gave the definition of the outgassing characteristic of the spacecraft's materials and summarized the rules and trends of outgassing of space materials. However, these researches cannot accurately predict the contamination, nor do they allow experimental verification. Numerical simulations of the contamination process in outgassing measuring experiments have been less well reported. Therefore, a more accurate dynamic simulation method of molecular contamination in an outgassing measuring system is demanded.

This study simulates molecular contamination in an outgassing measuring system. After dynamically simulating the outgassing deposition process, it measures the contaminant molecules from adhesive as the contamination source. It then analyzes the influence of the initial outgassing rate, diffusion coefficient and residence time on the simulation results. Based on the dynamics of several contamination species, it finally proposes methods for improving the accuracy of the simulated contamination process.

\section{NUMERICAL MODEL}

Outgassing experiments are usually performed in the free molecular flow regime. As the mean free path is much greater than the length scale of the vacuum chamber, the molecules collide with surfaces more frequently than with other particles. In this regime, we can ignore the intermolecular collisions and apply the angular coefficient method of COMSOL. This method computes the deposition by integrating the flux arriving at a surface from all other surfaces within its line-of-sight (not by the view factor between the outgassing surface and a sensitive surface). Therefore, this method greatly increases the calculation speed. 


\subsection{Geometry and Mesh Construction of the Vacuum Experiment}

To accurately simulate the test process, we build a geometric model based on ASTM E 1559 [18, 21], which comprises a vacuum test chamber, an outgassing unit, a vacuum pump, and three quartz crystal microbalance (QCM) detectors with the same view factor. The vacuum cabin is a cylinder of length $360 \mathrm{~mm}$ and radius $200 \mathrm{~mm}$. The outgassing unit is a cylinder of inner diameter $65 \mathrm{~mm}$ and depth $50 \mathrm{~mm}$. This unit has a cylindrical outgassing hole with an inner diameter equal to its depth $(3 \mathrm{~mm})$. The tubular vacuum pump (of inner diameter $140 \mathrm{~mm}$ ) operates at $500 \mathrm{l} / \mathrm{s}$. The normal to the three QCM detector faces passes through the center of the outgassing hole surface, with a length of $300 \mathrm{~mm}$ and a deviation angle of $10^{\circ}$ from the hole normal line.

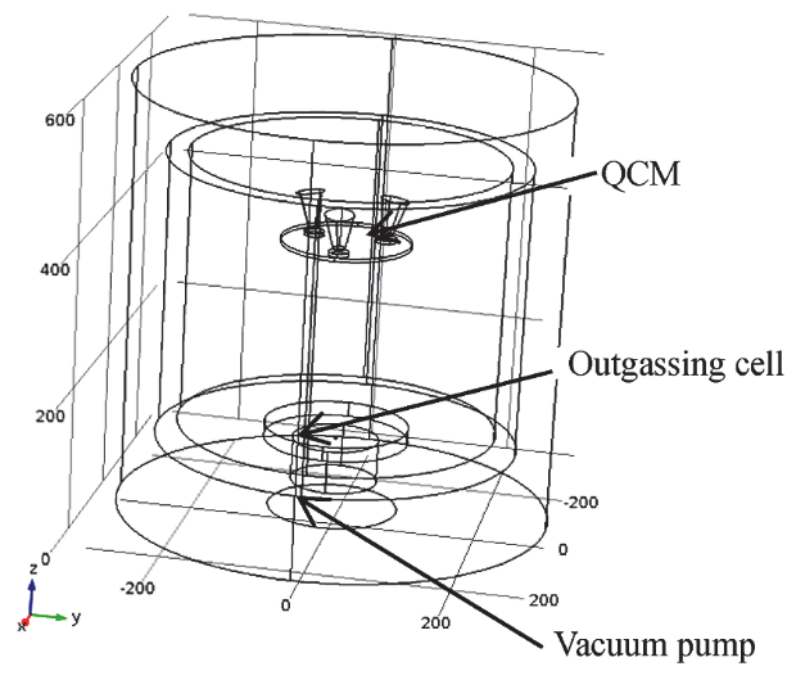

Figure 1 Computational model for experiment equipment

Fig. 2 shows the grid construct in the calculation model. The mesh is constructed by refining free tetrahedrons. To ensure an accurate calculation, the unit size of the deposition surface and outgassing hole is ranged from $0.08 \mathrm{~mm}$ to $1.5 \mathrm{~mm}$.

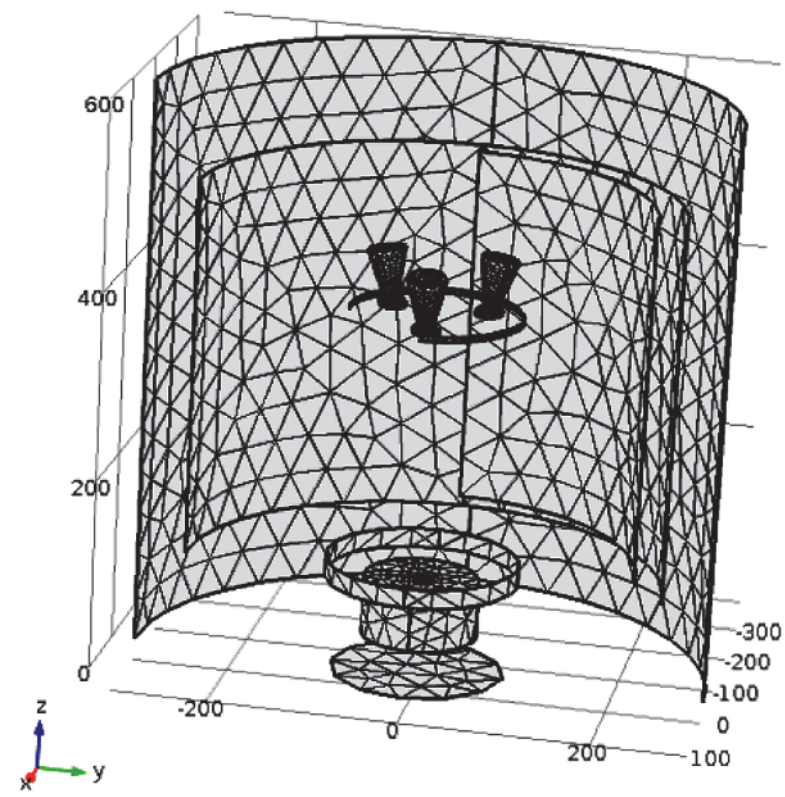

Figure 2 Schematic of experiment equipment computational grids
The contaminating molecules escape from the outgassing orifice and move inside the vacuum chamber. The initial velocity components parallel and perpendicular to the surface follow a Gaussian distribution and a biased Maxwell distribution, respectively. If a molecule reaches a chamber wall, it adsorbs to the surface; if it reaches the QCM, it is deposited on the surface with a certain probability, and is subsequently re-emitted from the surface with a certain probability.

\subsection{Outgassing Boundary Condition}

The outgassing orifice was set as the outgassing boundary in the outgassing model, which is based on diffusion theory $[16,20]$ :

$$
m=0.5 \cdot C_{0} \cdot d \cdot\left[1-\frac{8}{\pi^{2}} \exp \left(\frac{\pi^{2} \cdot D \cdot t}{d^{2}}\right)\right]
$$

where $m$ is outgassing mass, $C_{0}$ is initial concentration, $d$ is the thickness of sample, assumed to be $1 \mathrm{~mm}, D$ is diffusion coefficient. $C_{0}$ and $D$ are obtained based on ASTM E595 [17], its outgassing rate is as follow:

$$
\frac{\mathrm{d} m}{\mathrm{~d} t}=\frac{4 \cdot C_{0} \cdot D}{d} \exp \left(\frac{\pi^{2} \cdot D \cdot t}{d^{2}}\right)
$$

\subsection{Deposition Boundary Condition}

As the chamber wall in the experiment is cooled by liquid nitrogen, it attracts most of the contaminant molecules. Therefore, this wall was set as the adsorption wall with a sticking coefficient of 1.0. The surfaces of the three QCMs were set as the adsorption/desorption walls. In COMSOL, the simulated desorptions and adsorptions of the molecules are controlled by a molar desorption rate $\left(D_{1}\right)$ and a sticking coefficient $(S)$ :

$J=(1-S) G+D_{1} N_{\mathrm{A}}$

$\frac{\mathrm{d} n_{\mathrm{ads}}}{\mathrm{d} t}=\frac{S \cdot G}{N_{\mathrm{A}}}-D_{1}+\Gamma$

where $G$ is the incident molecular flux, $J$ is the emitted molecular flux, $N_{\mathrm{A}}$ is Avogadro's number, $n_{\text {ads }}$ is the molar concentration of adsorbed molecules, $\Gamma$ is an additional surface source of molar flux.

In this model the sticking coefficient $S$ is given the following value.

$$
S=S_{0} \cdot\left(1-\frac{n_{\text {ads }}}{n_{\text {sites }}}\right)
$$

where $S_{0}$ is sticking coefficient of a clean surface and is assumed to be $1, n_{\text {sites }}$ is the molar capacity of the surface sites on the system (assumed to be $1 \times 10^{-4} \mathrm{~mol} / \mathrm{m}^{2}$ ) $[11,12$, 19], the desorption rate is shown as follows: 
$D=\frac{n_{\mathrm{ads}}}{\tau}$

where $\tau$ is residence time. Eq. (6) assumes that the desorption process is first order.

$\tau=\tau_{0} \exp \left(\frac{E_{\mathrm{a}}}{R \cdot T}\right)$

where $E_{\mathrm{a}}$ is the chemical energy of desorption, $\tau_{0}$ is the lattice vibrational time (assumed to be $10^{-13} \mathrm{~s}$ ) in this model, we assume that the main contaminant species is water, so $\tau$ is assumed to be 1000s [11-13].

\section{EXPERIMENTS}

\subsection{Simulation Parameter Acquisition}

The parameters $C_{0}$ and $D$ were acquired in an apparatus used for measuring the effect of condensed outgassing contamination on a cryogenic sensitive surface in space [14]. The test materials were GD-414 and RTV566 silicone rubbers. The temperatures of the outgassing and deposition plate were $398 \mathrm{~K}$ and $90 \mathrm{~K}$, respectively. During the parameter test, the sample was heated evenly in a vacuum environment, and removed after $2 \mathrm{~h}$ and $4 \mathrm{~h}$ for weighing. The mass change of the sample before and after removal was divided by the heating time to obtain the average outgassing rate of the material in $1 \mathrm{~h}$ and $2 \mathrm{~h}$ respectively, the results are shown in Tab. 1.

\begin{tabular}{|c|c|c|}
\hline Material & $C_{0} / \mathrm{kg} / \mathrm{m}^{3}$ & $D / \mathrm{m}^{2} / \mathrm{min}$ \\
\hline RTV566 & 8.2 & $2.34 \times 10^{-10}$ \\
\hline GD-414 & 13 & $5.34 \times 10^{-10}$ \\
\hline
\end{tabular}

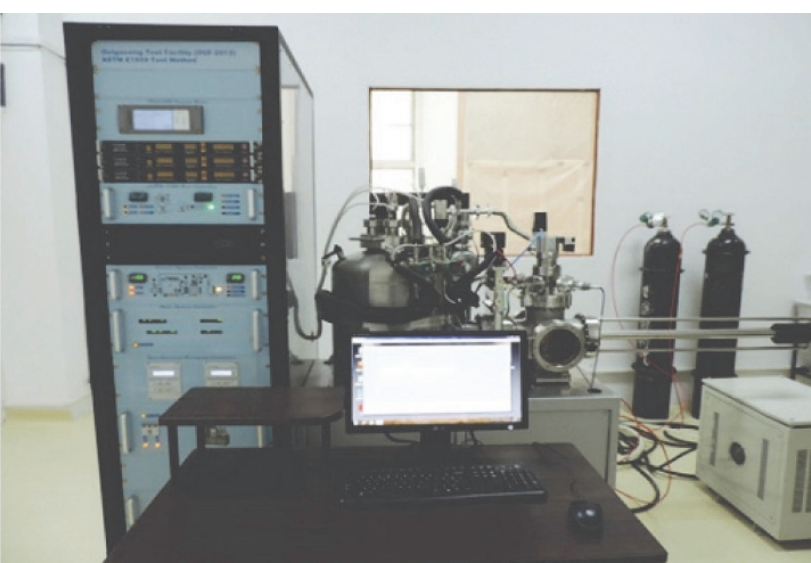

Figure 3 Laboratory equipment

\subsection{Verification Experiment}

The simulation results were verified on an integrated outgassing system (IOS-2013) designed to meet the requirements of the ASTM E1559 test. The IOS-2013 system typically includes a vacuum system, an internal outgassing/deposition measurement system, and a data acquisition system. The vacuum system consists of the main vacuum chamber, a liquid nitrogen system, and the associated vacuum chamber. Meanwhile, the internal outgassing/deposition measurement system contains the QCM device (with three QCM detectors) and an outgassing cell. The data acquisition system collects the QCM frequency, temperature data, temperature of the outgassing cell, and the time data (1 time datum/second). All tests were performed on RTV566 and GD-414 over several tens of hours. The temperature of the outgassing cell was fixed at $398 \mathrm{~K}$ while the QCM temperature was varied as $90 \mathrm{~K}$, $160 \mathrm{~K}$, and $298 \mathrm{~K}$.

\section{RESULTS}

\subsection{Verification}

Fig. 4 shows the calculation results of the RTV566 molecules outgassed at $398 \mathrm{~K}$ and deposited on the chamber cooled by liquid nitrogen. The colour scale in the two-dimensional deposition distribution represents the variation in the deposition mass from large (red) to small (blue). As seen in the diagram, the deposition mass first increased with height, and then decreased. The low deposition mass at the bottom of the chamber wall can be explained by blockage of the outgassing molecules by the outgassing cell. The deposition was highest in the middle of the chamber wall, where the view factor between the middle of the chamber wall and the outgassing hole was smallest. Therefore, the outgassing molecules could easily reach this part of the chamber. On the same deposition surface, the deposition mass should increase with increasing incident flow.

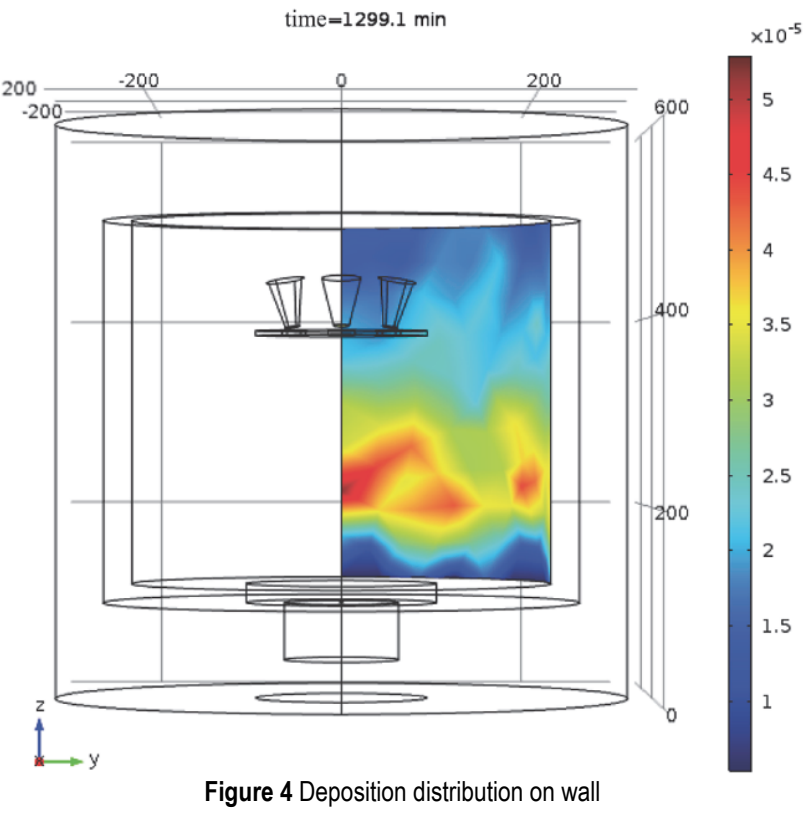

Fig. 5 shows the calculated outgassing molecular deposition on a sensitive $160 \mathrm{~K}$-surface generated by RTV566 at $398 \mathrm{~K}$. The uneven distribution of the deposition mass can be explained by the different view factors between the different positions on the deposition surface and the outgassing hole. When the deposition amount ranged from $2.5 \times 10^{-5} \mathrm{~kg} / \mathrm{m}^{2}$ to $4 \times 10^{-5} \mathrm{~kg} / \mathrm{m}^{2}$, the deposition masses at different positions on the deposition surface were very similar. Accordingly, the deposition was calculated as the average deposition per unit area.

Panels (a) and (b) of Fig. 6 compare the calculated and experimental data of contamination by RTV566 and GD414, respectively, where the outgassing and deposition temperatures were $398 \mathrm{~K}$ and $160 \mathrm{~K}$, respectively. In Fig. 
$6 \mathrm{a}$, the deposition mass increased and then stabilized. Before 100 minutes, the calculated and test data were consistent, but after 100 minutes, the model underestimated the test data. In Fig. 6b, the calculated and actual data were consistent until 300 minutes, and were obviously different thereafter. This discrepancy can be explained as follows. First, the simulation accounted only for the water molecules, and ignored other macromolecules. Initially, these small water molecules will be outgassed at a high rate, so the calculated deposition mass will fit the test results. At later outgassing stages, the outgassing rate of water molecules gradually decreases while the outgassing rate of large molecules begins to increase, so the test results exceed the calculation results. Second, the simulation parameters $C_{0}$ and $D$ were obtained from the average speed of particles in the chamber, and may differ from their actual values. To better simulate the outgassing of vacuum test materials, we must consider the influences of the initial outgassing rate, diffusion coefficient, and residence time on the simulation results, as discussed next.

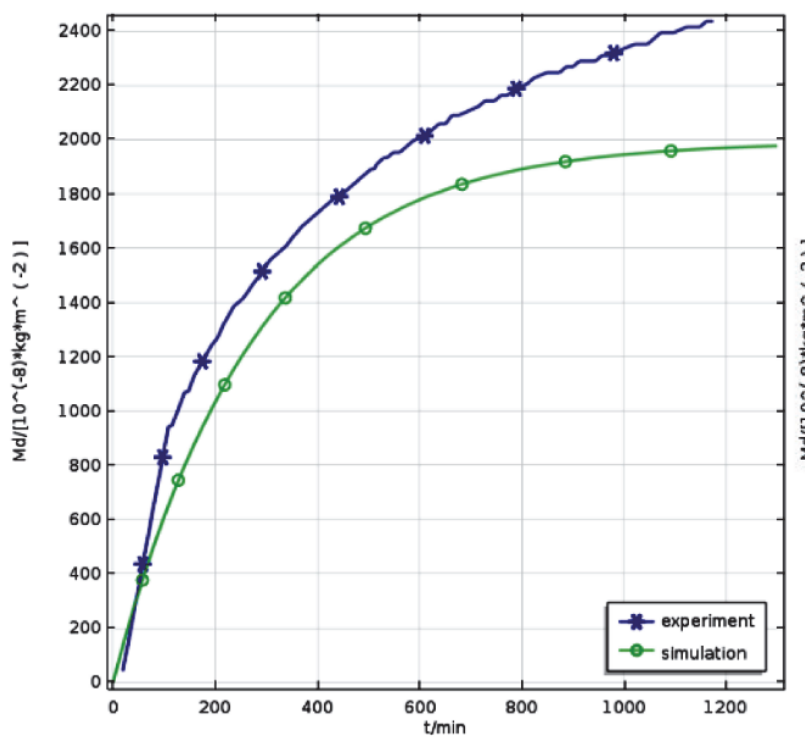

(a) RTV566

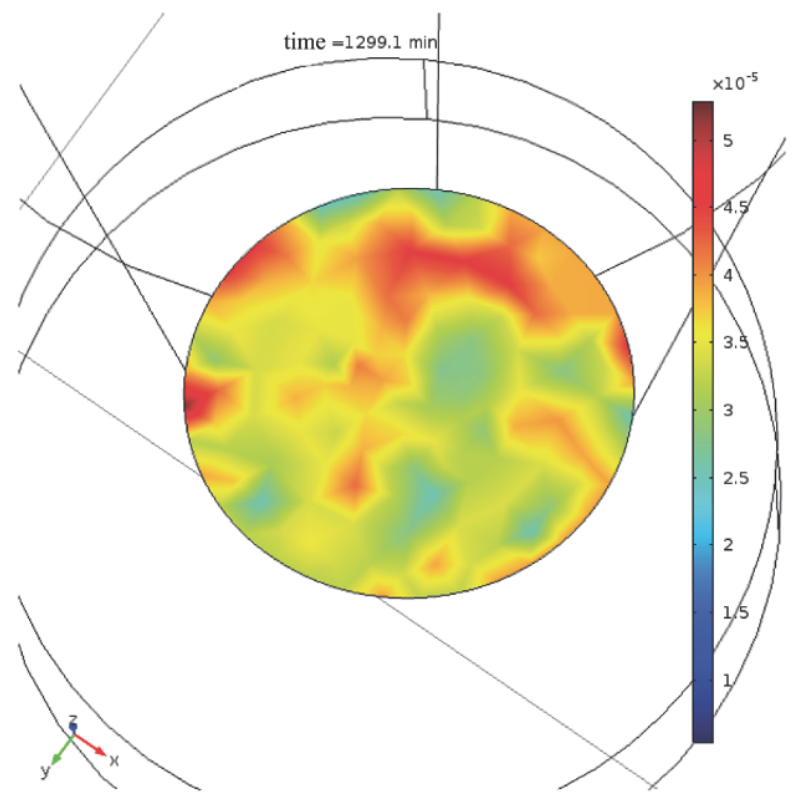

Figure 5 Deposition distribution on QCM

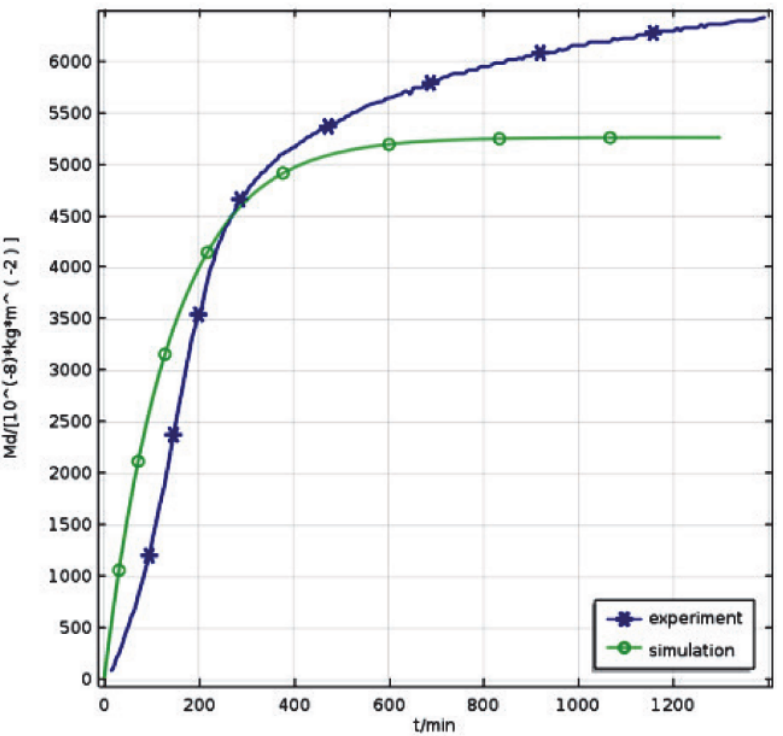

(b) GD414

Figure 6 Deposition distribution

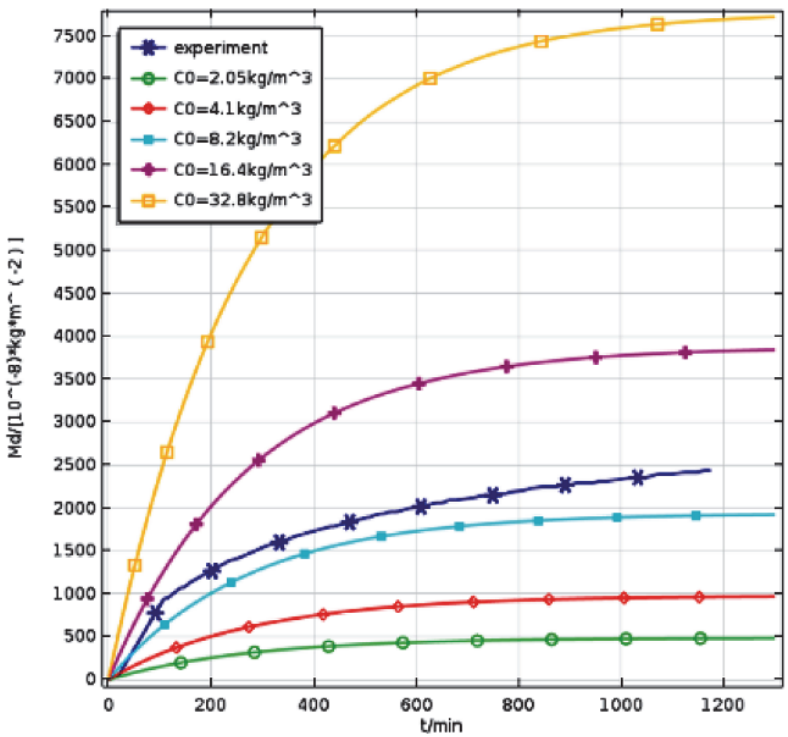

(a) RTV566

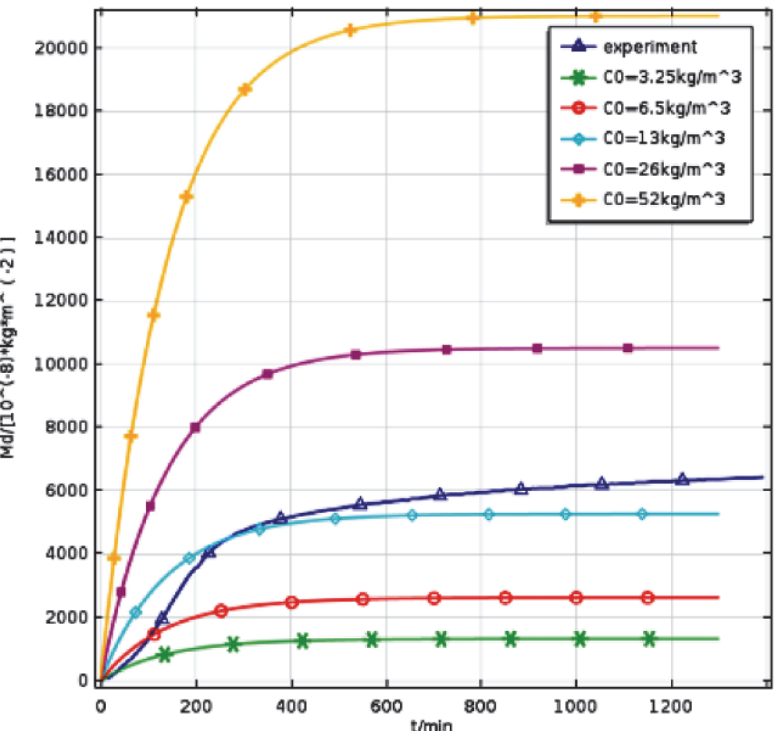

(b) GD414

Figure 7 Deposition distribution at different initial concentration 


\subsection{Analysis}

Panels (a) and (b) of Fig. 7 show the concentration dependences of the RTV566 and GD414 depositions, respectively. In Fig. 7a, the calculated and test results are consistent at the initial concentration $\left(8.2 \times 10^{-5} \mathrm{~kg} / \mathrm{m}^{3}\right)$, but between 4.1 and $16.4 \mathrm{~kg} / \mathrm{m}^{3}$, the difference between the test and calculated values extends to $1.5 \times 10^{-5} \mathrm{~kg} / \mathrm{m}^{3}$, and the gap widens at higher concentrations. As the concentration of the expelled contaminant increases, the outgassing rate will increase, so the mass and rate of the deposition rate will both increase. In other words, increasing the molecular incidence rate will enhance the deposition mass and deposition rate on the same deposition surface. Similar trends are observed in Fig. $7 \mathrm{~b}$.

Panels (a) and (b) of Fig. 8 show the diffusion coefficient dependences of the RTV566 and GD414 depositions, respectively. In Fig. 8, the calculated and test results are consistent at the diffusion coefficient which is obtained from the test, in Fig. 8a, at the diffusion coefficient of $9.36 \times 10^{-10} \mathrm{~m}^{2} / \mathrm{min}$, the deposition mass is 7 times of the test data. increasing the diffusion coefficient will enhance the deposition mass and deposition rate on the same deposition surface. The reason is similar to the effect mechanism of initial concentration.

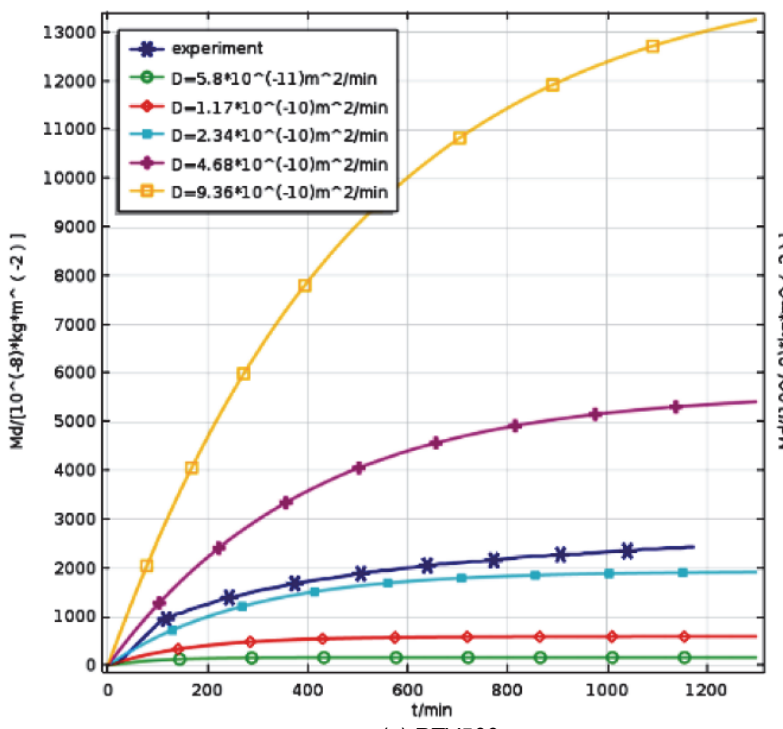

(a) RTV566

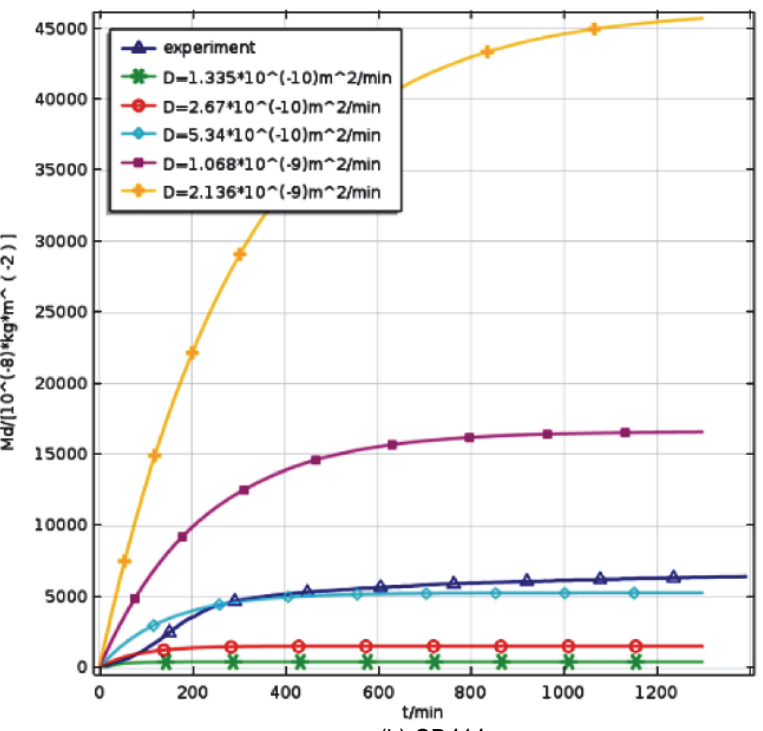

(b) GD414

Figure 8 Deposition distribution at different diffusion coefficient

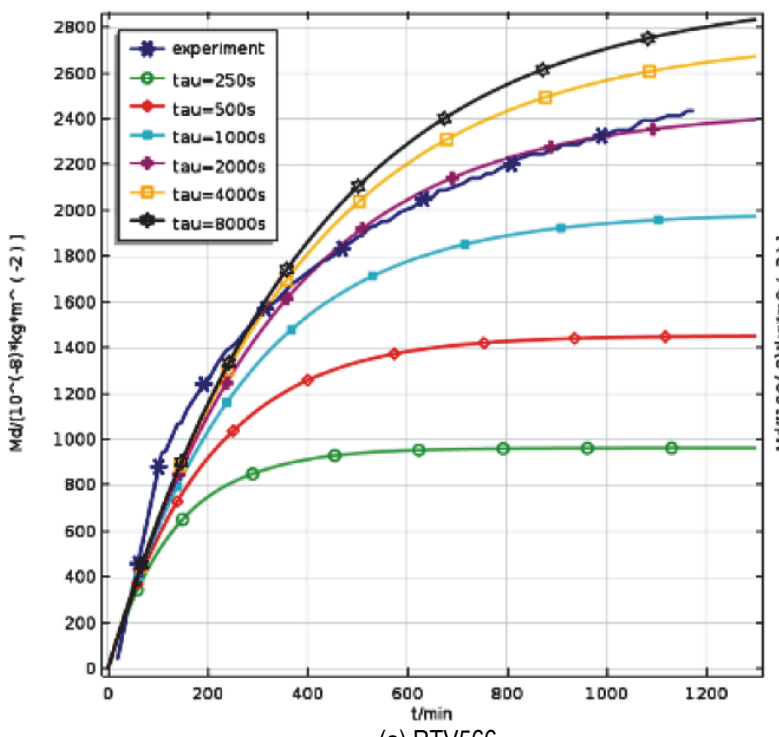

(a) RTV566

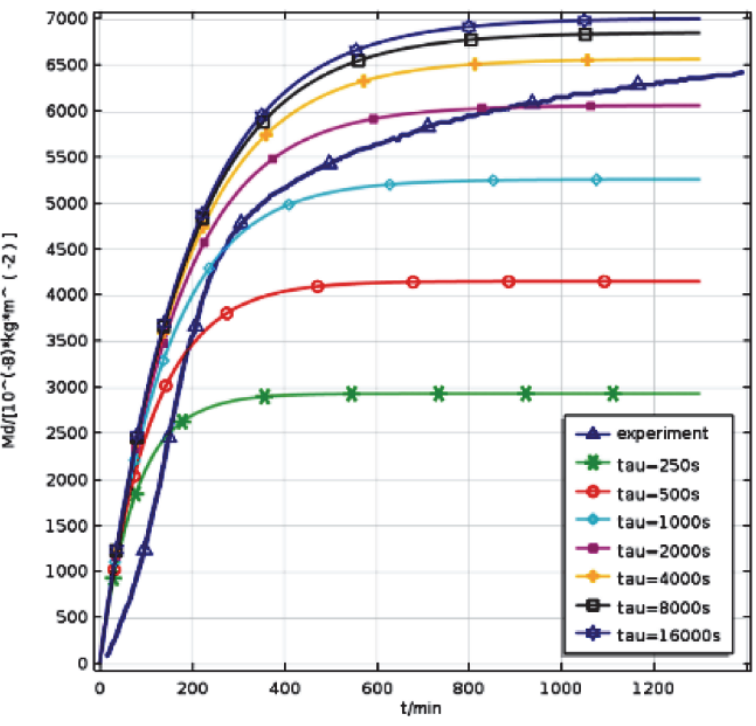

(b) GD414

Figure 9 Deposition distribution of different desorption time constant

Panels (a) and (b) of Fig. 9 show the residence time dependences of the RTV566 and GD414 depositions, It can be seen from the figure that when the residence time is 250 s, $500 \mathrm{~s}, 4000 \mathrm{~s}$ and $8000 \mathrm{~s}$, the gap widens. however calculation results are close to the test data at the residence time of $1000 \mathrm{~s}$ and $2000 \mathrm{~s}$. It can be inferred that the deposition molecules of RTV566 contain macromolecules with residence time of $2000 \mathrm{~s}$.

\subsection{Improvement}

Based on the above analysis, we infer that when the diffusion coefficient and the initial concentration are obtained from the test data, the calculation result will better match the test data. The parameter choice greatly affects the simulation results. To improve the calculation method, we focused on the residence time. As shown in Fig. 9, the 
residence time of macromolecular contamination by the two materials is $2000 \mathrm{~s}$ and $1000 \mathrm{~s}$. Considering both macromolecules and water molecules in the simulation, at

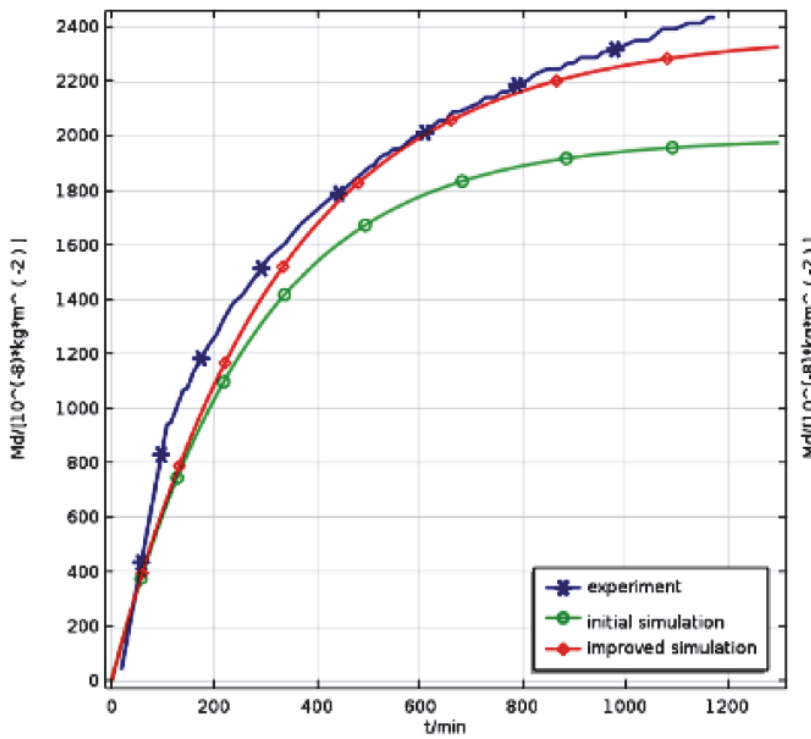

(a) RTV an assumed mass ratio of 1:1, the calculation results of both RTV and GD414 were vastly improved from the initial simulation results (Fig.10a and 10b, respectively).

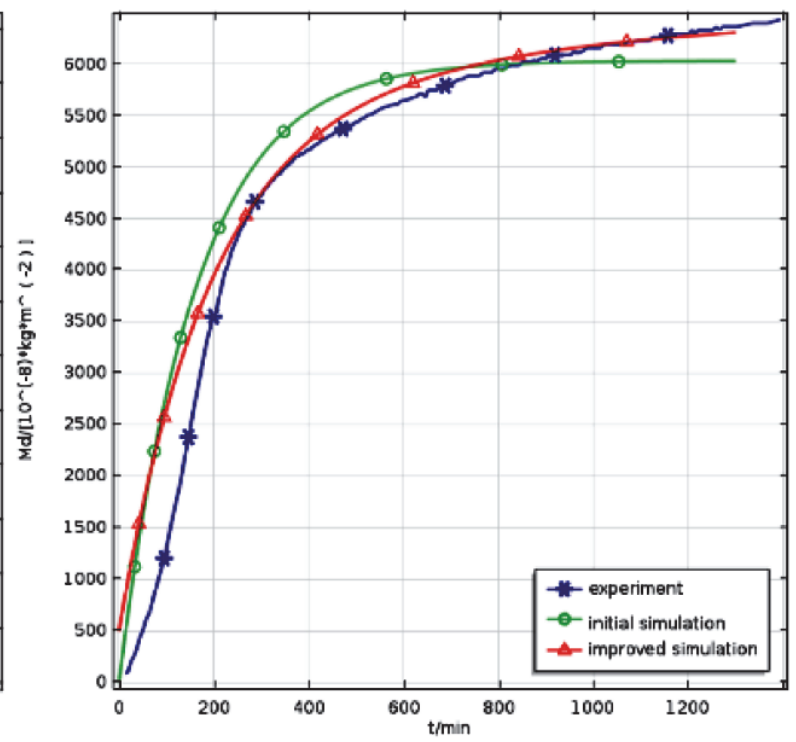

(b) GD414

Figure 10 Deposition distribution

\section{CONCLUSIONS}

We use the improved analytic method to simulate the molecular contamination transport process dynamically and measure the contaminant molecules from adhesive as the contamination source. Then we analyze the impact of the initial outgassing rate, diffusion coefficient and residence time on the simulation results and improve the accuracy based on the dynamics of several contamination species finally.

The main conclusions of the study are summarized below.

(1) The angle coefficient method can calculate the molecular outgassing contamination process of materials in a high-vacuum environment.

(2) The two-molecular species model effectively improved the numerical precision of the simulated outgassing contamination process in a high vacuum environment. The outgassing molecules of different materials differ mainly by their chemical energies of desorption. Therefore, the multi-species simulation is expected to improve numerical simulations of the outgassing contaminants of materials other than those investigated here.

(3) A precise simulation calculation requires the accurate mass ratios and desorption chemical energies of the outgassing molecules. To this end, we must establish test methods and test equipment that accurately determine the types of components, their mass ratios, and their chemical energies of desorption.

(4) The initial concentration and diffusion coefficient largely influence the numerical results of material outgassing contamination. Therefore, to accurately simulate the molecular contamination process, we must more accurately determine the initial concentration and diffusion coefficient parameters, which require improved test methods and equipment.
So in order to acquire more accurate results, we must establish test methods and test equipment that determine the types of components, their mass ratios, their chemical energies of desorption and so on.

\section{Acknowledgement}

This paper is supported by the Equipment pre-research key laboratory fund (6142207190203).

\section{REFERENCES}

[1] ASTM Designation: E 1559-09 (2016). Standard test method for contamination outgassing characteristics of spacecraft materials.

[2] Anderson, J. R., Ferraro, N., \& Soares, C. (2018). Implications of New Stringent Requirements for Contamination Control. Proceedings of SPIE, 10748, 1-3.

[3] White, L. (2017). Organic and inorganic contamination control approaches for return sample investigation on Mars 2020. 2017 IEEE Aerospace Conference, 1-10. https://doi.org/10.1109/AERO.2017.7943709

[4] Heske, A. (2016). Science and payloads for the next decades of ESA's Cosmic Vision program. 2016 IEEE Aerospace conference, 1-13. https://doi.org/10.1109/AERO.2016.7500853

[5] Brockewll, T. G. (2016). The Mass Spectrometer for Planetary Exploration (MASPEX). 2016 IEEE Aerospace Conference, 5-12. https://doi.org/10.1109/AERO.2016.7500777

[6] Bieler, A. (2016). Mass spectrometric characterization of the Rosetta Spacecraft contamination with ROSINA. Proceedings of SPIE, 9952, 1-5.

[7] Wong, C. M., Labatete-Goeppinger, A. C., Fowler, J. D. et al. (2016). Outgassing Study of Spacecraft Materials and Contaminant Transport Simulations. Proceedings of SPIE, 9952, 3-12. https://doi.org/10.1117/12.2239103

[8] Silver, D. M. (2001). Midcourse Space Experiment: Early Flight Molecular Contamination Modeling Predictions. 35th Aerospace Sciences Meeting \& Exhibit, AIAA 97-0839.

[9] Huang, S. \& Hetrick, M. A. (1986). Preliminary Correlation of Spacecraft Contamination Flight Data with the Modified 
SPACE II Computer Model. AIAA/ASME 4th Thermophili's and Heat Transfer Conference, AIAA-86-1357. https://doi.org/10.2514/6.1986-1357

[10] Khassanchine, R. H., Grigorevskiy, A. V., \& Galygin, A. N. (2004). Simulation of Outgassing Processes in Spacecraft Coatings Induced by Thermal Vacuum Influence. Journal of Spacecraft Rocket, 41(3), 384-389. https://doi.org/10.2514/1.10933

[11] Jarossy, F. J., Pizzicaroli, J. C., \& Ress, E. B. (1980) Shuttle/Payload Contamination Evaluation (SPACE) - A Systems Level Contamination Model. Proc. SPIE 0216, Optics in Adverse Environments II, 13-22. https://doi.org/10.1117/12.958444

[12] Giunta, I., Lemcke, C., Roussel, J. F. et al. (2000) Osean/COMOVA version 1.0 contamination modelling outgassing / vent analysis tool final report.

[13] Yuan, X. X., Pang, H. W., \& Zhuou, C. L. (2007). Study on attenuation of solar cell wing power by molecular pollution. Journal of Astronautics, 28(1), 118-122.

[14] Jin, X. H., Huang, F., Cheng, X. L. et al. (2015). Test particle Monte Carlo simulation for return flux due to ambient scatter of outgassing molecules on a circular disk. Acta Aerodynamica Sinica, 33(6), 782-798. https://doi.org/10.1063/1.4902713

[15] Shuang, W. (2018). Research on pollution evaluation method of space optical system. Harbin Institute of Technology, Harbin.

[16] Yao, R. J., Wang, Y., Wang, X. R. et al. (2007). Application of Outgassing Model of Nonmetal Materials for Satellite. Aerospace Material Technology, 33(2), 48-50.

[17] ANSI/ASTM E-595. Standard Test Method for Total Mass Loss and Collected Volatile Condensable Materials from Outgassing in a Vacuum Environment.

[18] O'Hanlon, J. F. (2003). A User's Guide to Vacuum Technology. Nuclear Technology, 55(3), 732-733. https://doi.org/10.13182/NT81-A32825

[19] Dylla, H. F. (2006). The Problem of Water in Vacuum Systems. CERN Accelerator School, Platjad'Aro, Spain.

[20] Lafferty, J.M. (1998). Foundations of Vacuum Science and Technology. IEEE Electrical Insulation Magazine, 14(4), 42-42. https://doi.org/10.1109/MEl.1998.689278

[21] Wang, X. R. \& Ma, W. J. (2002). A simulation equipment used for determining the outgassing contamination condensation effect on cryogenic sensitive surface in space. Journal of Astronautics, 23(3), 68-71.

\section{Contact information:}

Jia QIAO

(Corresponding author)

Lanzhou Institute of Physics,

Lanzhou, Gansu, 730000, China

E-mail: qiaojia_lip@163.com

\section{Shengsheng YANG}

Lanzhou institute of Physics,

Lanzhou, Gansu, 730000, China

\section{Jianjun LI}

Lanzhou institute of Physics,

Lanzhou, Gansu, 730000, China

\section{Xing GUO}

Lanzhou institute of Physics,

Lanzhou, Gansu, 730000, China

\section{Yi WANG}

Lanzhou institute of Physics,

Lanzhou, Gansu, 730000, China 